Elsevier

BPC 00880

\title{
FORWARD RATE CONSTANTS FOR RECEPTOR CLUSTERS VARIATIONAL METHODS FOR UPPER AND LOWER BOUNDS
}

\author{
J.H.J. VAN OPHEUSDEN ${ }^{\text {a }}$, F.W. WIEGEL a and B. GOLDSTEIN ${ }^{\text {b }}$ \\ a Depariment of Applied Plysics, Twente University of Technology P.O. Box 217, 7500 AE Enschede, The Netherlands and b Los \\ Alamos National Laboratory, University of California, Los Alamos, NM 3754.7. U.S.A.
}

Received 22nd December 1983

Accepted 8th May 1984

Key words: Forward rate constant; Receptor cluster; Variational principle; Electrostatic analogy; Chemoreception

\begin{abstract}
We are interested in the effect of receptor clustering on $k_{+}$, the diffusion-limited forward rate constant for the binding of a ligand to a cell surface receptor. Here we estimate the reduction in $k_{+}$when receptors are clustered in various configurations. We obtain two alternative expressions for the flux of ligands into receptors distributed on a surface. Next we show through a variational principle that these provide both upper and lower bounds on the flux when evaluated for trial concentration functions which satisfy only the boundary conditions of the Laplace equation. We use an analogy with electrostatics to calculate rigorous bounds within approx. $10 \%$ of the exact result for a variety of planar clusters of hemispherical receptor sites. We also obtain an exact result for the flux into a spheroidal receptor and use this result to obtain bounds on the flux into certain receptor clusters.
\end{abstract}

\section{Introduction}

In order for cells to sense what is happening about them, they must set up a flow of data from their environment into their interior. One major source of such information begins with the detection of ligands, such as polypeptide hormones, neurotransmitters, transport proteins, and antigens, that follow diffusive paths through the surrounding fluid to the cell surface. Embedded in the plasma membrane of the cell, but usually capable of translational and rotational motion, are a variety of different receptor molecules. Each different receptor is highly selective in its binding properties and is usually capable of combining only with a single ligand or a very limited number of closely related ligands.

At first glance the diffusive nature of information flow from ligand in solution to cell surface receptor may seem inefficient. However, it is exactly the diffusive character of the flow that ena- bles a cell to detect a ligand efficiently even though it has relatively few receptors specific for the ligand on its surface. With just several thousand receptors covering only $0.1 \%$ of the cell surface, the ligand can be detected with almost maximum efficiency $[1,2]$. Thus, a cell can efficiently detect approx. 100 different ligand species by dispersing over the cell surface several thousand receptors for each ligand.

For a few simple idealized geometries of the cell-receptor-ligand system, there exist exact analytical results for the total flux of ligands into the receptor, given the ligand concentration far from the cell and the ligand's transport coefficient in the medium. For example, when the cell surface is treated as a reflecting surface and the receptor as an absorbing hemisphere of radius $a$, the flux into the receptor equals $2 \pi a D c_{\infty}$ [3], just half the result obtained by Smoluchowski [4], where $D$ is the diffusion coefficient of the ligand in the medium and $c_{\infty}$ the ligand concentration far from the receptor. When the receptor is treated as a flat, 
perfectly absorbing disk of radius $a$, the flux equals $4 a D c[1,2]$, somewhat less than that into a hemisphere of the same radius. These results hold in the limit when the receptors are infinitely dilute on the cell surface.

For the case of a large number of receptors of simple geometry. distributec randomly across a roughly spherical cell membrane, there exist approximate solutions that explain many of the salient features of diffusion-limited receptor binding to cells. Often, however, the binding sites on the cell surface are clustered rather than randomly distributed. For example, the insulin receptor is aggregated on certain cell types [5]. In the absence of the ligand, the low-density lipoprotein receptor on human fibroblasts is clustered in coated pits [6], and the epidermal growth factor receptor may also be clustered on these cells [7]. All cells that have antibody on their surface have clustered binding sites, since all cell surface antibodies are bivalent [8]. The acetylcholine receptor also appears to have two separate binding sites for cholinergic ligands [9].

In this paper we consider the effect of receptor clustering on the diffusion-limited forward rate constant. We start by deriving two alternative expressions for the diffusive flux into receptors on a cell surface, in terms of the concentration profile $c(\bar{r})$ of the ligand under consideration. This profile may be found by solving the Laplace equation for $c$ with boundary conditions that describe the effect of the cell membrane and the receptor binding sites. For most cases of interest, unfortunately, it is very difficult, if not impossible, to lind exact analytic solutions of Laplace's equation. However, we will show through a variational principle that the two expressions we derived for the diffusive flux can be used to calculate rigorous upper and lower bounds on the exact forvard rate constant for arbitrary surface geometries. This is done by considering trial concentration profiles that satisfy the boundary conditions but not necessarily Laplace's equation itself. Thus, this paper forms a generalisation of the work of Goldstein and Wiegel [3], who discussed only the upper bound formalism. In sections 2 and 3 we discuss the variational formalism for obtaining upper and lower bounds, respectively. In section 4 we invoke the analogy between this theory and the Dirichlet and Thomson bounds in electrostatics to propose two fairly simple trial distributions to set bounds on the forward diffusive flux into a number of non-intersecting hemispherical receptor sites in a planar membrane. Then, in section 5 we evaluate these bounds for a variety of such clusters. Also, we use the electric analogy to calculate the exact flux into a spheroidal receptor. For certain gcometrics of clustered receptors, we exploit this result to obtain improved upper and lower bounds on the flux.

\section{Upper bounds}

We wish to calculate the inward diffusive flux $J$ for a cell with a number of receptors distributed along its outer membrane. The ligand concentration $c(\vec{r})$ far from the receptors is taken to be constant, while at the receptors we assume a complete and instantaneous absorption. Thus $c$ should be zero there. Furthermore, we will assume that the membrane itself simply reflects the ligands, which implies that the concentration gradient $\vec{\nabla} c$ must be parallel to the surface. If there are no sources or other sinks of ligands in the rest of the fluid we must solve

$\Delta c=0$

with boundary conditions $c=0$ at the receptors, the normal derivative $\partial c / \partial n=0$ at the membrane and $c=c_{\infty}$ far away.

The local current density is

$\vec{J}=-D \vec{\nabla} c$

where $D$ is the translational diffusion constant for the ligands in the plasma outside the cell. The total inward flux into the cell is found by integrating $\bar{J}$ over the surface of the binding sites

$J=J_{\mathrm{o}} \equiv D \rho \vec{\nabla} c \cdot \overrightarrow{\mathrm{AS}}$

Here $\overrightarrow{\mathrm{d} S}$ is an infinitesimal surface element of the receptors with outward pointing unit vector $\hat{\boldsymbol{n}}$. As we have taken the boundary condition $\partial c / \partial n=$ $\vec{\nabla} c \cdot \hat{n}=\mathbf{0}$ at the membrane we may as well extend the integration in eq. 3 over the whole cell surface, both membrane and receptors. 
We now convert eq. 3 into a form that is more suitable for our purposes. Consider therefore a closed surface $\partial V_{\infty}$, sufficiently far away from the cell surface $\partial V_{0}$ that we may take the bulk concentration $c_{\infty}$ to be reached all over $\partial V_{\infty}$ (fig. 1 ). Because we have conservation of ligands we may evaluate eq. 3 over $\partial V_{\infty}$. As $c$ is constant on $\partial V_{\infty}$ we multiply by $c / c_{\infty}$ and take $c$ under the integral sign to obtain the cquivalcnt expression

$J=-D / c_{\infty} \int_{\partial \nu_{x}} c \vec{\nabla} c \cdot \overrightarrow{\mathrm{d}} \overrightarrow{\mathrm{S}}$

where the minus sign appears because we take $\overrightarrow{\mathrm{d}} \overrightarrow{\boldsymbol{S}}$ to be pointing inward.

The same expression, when evaluated over $\partial V_{0}$, the cell surface, yields zero, as either $c$ or $\vec{\nabla} c \cdot \hat{n}$ is zero. By application of Gauss' theorem and using eq. 1 we now transform eq. 3 into

$J=J_{1} \equiv \frac{D}{c_{\infty}} \int_{\nu}(\vec{\nabla} c)^{2} \mathrm{~d} V$

Letting $\partial V_{\infty}$ go to infinity the volume $V$ becomes just the exterior of the cell.

The virtue of the formalism according to eq. 5

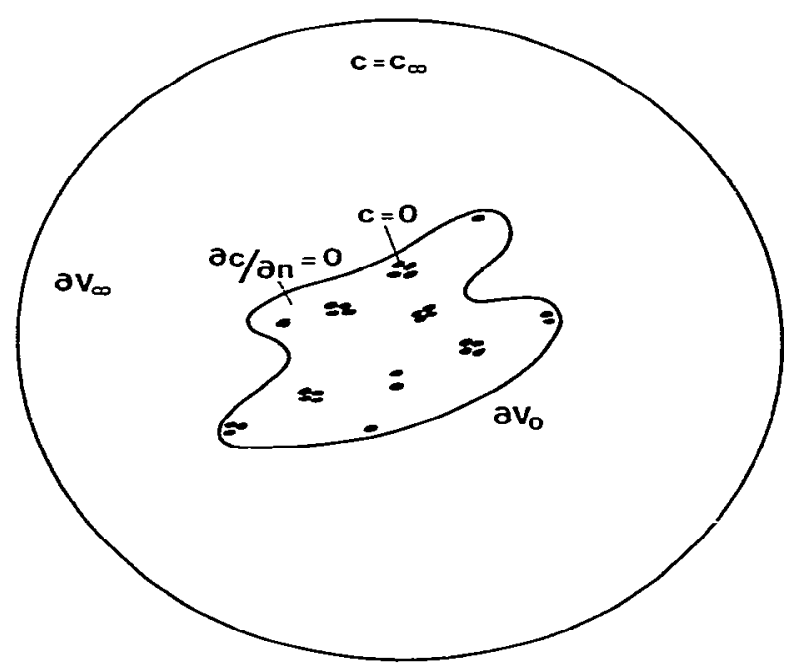

Fig. 1. The general geometry of a cell surface $\partial V_{0}$, consisting of a membrane reflecting ligands $(\partial c / \partial n=0)$ with embedded receptors $(c=0)$. The ligand concentration $c_{\infty}$ at a surface $\partial V_{\infty}$ far away is taken to be constant. is that this expression allows for the existence of a variational principle: $J_{1}[c]$ for functions $c$ that satisfy the boundary conditions is minimized for $\Delta c=0$. So, given a certain boundary value problem we may take a trial function $c_{1}$, evaluate $J_{1}\left[c_{1}\right]$ and conclude that

$J_{1}[c] \leq J_{1}\left[c_{1}\right]$

where $c$ is the exact solution. Thus, we obtain a rigorous upper bound on the exact flux without the need to solve the full eq. 1 .

That $J_{1}[c]$ indeed forms a minimum is proven as follows. Consider a variation $\epsilon \rho(\vec{r})$, where $\epsilon$ is small, around some function $c$ satisfying the boundary conditions of eq. 1 . We find

$$
\begin{aligned}
J_{1}[c & +\epsilon \rho]=\frac{D}{c_{\infty}} \int_{V}(\vec{\nabla} c+\epsilon \vec{\nabla} \rho)^{2} \mathrm{~d} V \\
= & \frac{D}{c_{\infty}}\left[\int_{V}(\vec{\nabla} c)^{2} \mathrm{~d} V+2 \epsilon \int_{V}(\vec{\nabla} c \cdot \vec{\nabla} \rho) \mathrm{d} V\right. \\
& \left.+\epsilon^{2} \int_{V}(\vec{\nabla} \rho)^{2} \mathrm{~d} V\right] .
\end{aligned}
$$

The coefficient linear in $\epsilon$ is called the first variation. Using Gauss' theorem and the vector equation $\bar{\nabla} \cdot(\rho \bar{\nabla} c)=\rho \Delta c+\bar{\nabla} \rho \cdot \bar{\nabla} c$, we write it in the form

$\int_{V} \vec{\nabla} \rho \cdot \bar{\nabla} c \mathrm{~d} V=\int_{V} \rho \Delta c \mathrm{~d} V+\int_{\partial V_{0}+\partial V_{x}} \rho \bar{\nabla} c \cdot \overrightarrow{\mathrm{d} S}$

Now $\rho=0$ at the receptors and at infinity, while at the membrane $\partial c / \partial n=0$, so we conclude that the second integral on the right-hand side yields zero. It follows that $J_{1}[c]$ is stationary if and only if $\Delta c=0$. Because the contribution to second order in $\epsilon$ is positive definite the stationary point forms a minimum.

From eq. 5 we see that under scale transformations $J_{1}[\beta c]=\beta J_{1}[c]$. The definition

$J_{1}\left[c_{1}\right]=D c_{\infty} \int_{V}\left(\frac{\vec{\nabla} c_{1}}{c_{1, \infty}}\right)^{2} \mathrm{~d} V$

is invariant for scale transformations of the trial function $c_{1}$. Hence, a constant but nonzero $\rho$ at infinity is simply scaled away, meaning that the boundary conditions may be somewhat relaxed. In 
order to have a finite, nonzero $J$, however, we must ensure that $\int_{\partial v_{\infty}} \vec{\nabla} \rho \cdot \overrightarrow{\mathrm{d}}$ is finite, so $\left|\vec{\nabla}_{\rho}\right|-r^{-2}$ as $r \rightarrow \infty$. In terms of the diffusion-limited forward rate constant, $k_{+}=J / c_{\infty}$, we have

$k_{+} \leq \frac{D}{c_{\infty}^{2}} \int_{V}(\bar{\nabla} c)^{2} \mathrm{~d} V$

for trial functions $c$ satisfying the boundary conditions at the cell surface and $\mid \vec{\nabla} c] \sim r^{-2}$ as $r \rightarrow \infty$.

Note that nowhere in this formalism have we made explicit use of the form of the receptors or the membrane surface, apart from the fact that we have assumed the integrals to exist and be convergent. Thus, these expressions are valid for arbitrary surface geometry of the cell and its receptors. In this sense this paper forms a generalisation of the work of Goldstein and Wiegel [3].

One immediate corollary of this is that given a certain geometry $A$ we can consider a cell geometry $B$ such that the receptors of $A$ lie completely within those of $B$. If we take the trial function $c_{1}$ for the flux $J_{\mathrm{A}}$ to be the solution for case $\mathrm{B}$ and zero in the region in between we have

$J_{\mathrm{A}} \leq J_{\mathrm{B}}$

Physically this can be understood because a ligand crossing the virtual receptor surface of $B$ may diffuse away and need not contribute to $J_{\mathrm{A}}$. Although the result (eq. 11) is obtained in a rather trivial way, the cases in which geometry $B$ is such that there exist exact analytical results for $J_{\mathrm{B}}$ may yield quite nontrivial upper bounds for $J_{\mathrm{A}}$. On physical grounds one may also conclude that upon reversal of the roles of $A$ and $B$ in the last discussion eq. 11 can be used to derive a lower bound on $J_{\mathrm{B}}$.

It would, however, be desirable to have a more general formalism like the one we have described for the upper bound. Indeed, there exists a function for $J$ complementary to that developed in this section in the sense that its stationary point forms an absolute maximum. This will then yield lower bound estimates for the diffusion-limited flux. We shall derive this complementary expression and give a detailed description of the formalism in section 3 .

\section{Lower bounds}

With eqs. 3 and 5 we have two equivalent, mathematically independent expressions for the ligand flux $J$ into a cell, given the concentration profile $c(\vec{r})$ and its gradient. We then have a whole class of expressions by considering linear combinations of these. One that will be particularly useful is

$$
\begin{aligned}
J & =J_{2} \equiv 2 J_{0}-J_{1} \\
& =\frac{D}{c_{\infty}}\left[2 \int_{\partial V_{0}} c_{\infty} \vec{\nabla} c \cdot \overrightarrow{\mathrm{d} \vec{S}}-\int_{V}(\vec{\nabla} c)^{2} \mathrm{~d} V\right]
\end{aligned}
$$

Again we consider variations $\epsilon \rho$ around $c$, such that $c$ and $c+\epsilon \rho$ meet the boundary conditions. The variation is

$$
\begin{aligned}
\delta J_{2}= & \frac{2 D \epsilon}{c_{\infty}}\left[\int_{\partial V_{0}} c_{\infty} \vec{\nabla} \rho \cdot \overrightarrow{\mathrm{d}} \vec{S}-\int_{\partial V_{0}+\partial V_{\infty}} \rho \bar{\nabla} c \cdot \overrightarrow{\mathrm{d}} \vec{S}\right. \\
& \left.+\int_{V} \rho \Delta c \mathrm{~d} V\right]-\frac{D \epsilon^{2}}{c_{\infty}} \int_{V}(\vec{\nabla} \rho)^{2} \mathrm{~d} V
\end{aligned}
$$

The second integral in this expression is zero again. In order that $J_{2}$ be stationary, we must impose two conditions

$\Delta c=0$

and

$\int_{\mathrm{a} \nu_{0}} \vec{\nabla} \rho \cdot \overrightarrow{\mathrm{d}} \overrightarrow{\mathrm{S}}=0$

Thus, we must restrict ourselves to variations of the distribution of the inward flux $\bar{j}$ over the receptor surface, fixing $J_{0}$. As this will modify the corresponding flux in the volume $V, J_{1}$ will change accordingly. Note that the solution of the Laplace equation with a given set of boundary conditions is unique. We will show shortly how this restriction may be relaxed.

The second variation is negative definite and $J_{2}$ is maximal at its stationary point: we have

$J_{2}\left[c_{2}\right] \leq J_{2}[c]$

for any trial function $c_{2}$.

Note, however, that if we consider a scale transformation $c_{2} \rightarrow \beta c_{2}$ and maximize eq. 12 for 
$\mathrm{d} J_{2} / \mathrm{d} \beta=0$ we obtain the alternative form

$J_{2}=D c_{\infty} \frac{\left(\int_{\partial V_{0}} \vec{\nabla} c \cdot \overrightarrow{\mathrm{d}} \overrightarrow{\boldsymbol{S}}\right)^{2}}{\int_{V}(\vec{\nabla} c)^{2} \mathrm{~d} V}$

A general variation $c \rightarrow c+\epsilon \rho$ can be thought of as being composed of two successive steps. First, a scale transformation $c \rightarrow c^{\prime}$, where

$c^{\prime}=c\left[\frac{\int(\bar{\nabla} c+\epsilon \vec{\nabla} \rho) \cdot \overrightarrow{\mathrm{d}} \vec{S}}{\int \bar{\nabla} c \cdot \overrightarrow{\mathrm{d}} \overline{\boldsymbol{S}}}\right]$

This does not influence the flux $J_{2}$ as calculated through the scale invariant expression (16). The second step consists of $c \rightarrow c^{\prime}+\epsilon \rho^{\prime}=c+\epsilon \rho$, where now $\int_{a \nu_{0}} \vec{\nabla} \rho^{\prime} \cdot \overrightarrow{d S}=0$, in accordance with eq. $14 \mathrm{~b}$. This implies that the restriction eq. $14 \mathrm{~b}$ can indeed be dropped when one uses eq. 16 for the flux. Note that a scale transformation such as eq. 17 does violate the boundary condition at infinity, but for eq. 16 to form a maximum we only require that $J_{2}[\beta c]$ forms a maximum when interpreted as a function of $\beta$. Readers interested in the mathematical details of the formalism are referred to the book of Arthurs [11].

Again we have not made use of any details of the geometry. Hence, our result holds for cells of arbitrary shape with an arbitrary distribution of receptors. For any given geometry we can place rigorous upper as well as lower bounds on the diffusion-limited flux by considering trial functions $c_{1}$ and $c_{2}$.

Eqs. 6 and 15 then give

$J_{2}\left[c_{2}\right] \leq J[c] \leq J_{1}\left[c_{1}\right]$

where for the exact solution $c$ the two definitions coincide. In terms of the diffusion-limited forward rate constant we have

$D \frac{\left(\int_{\partial V_{0}} \vec{\nabla} c_{2} \cdot \overrightarrow{\mathrm{d}}\right)^{2}}{\int_{V}\left(\vec{\nabla} c_{2}\right)^{2} \mathrm{~d} V} \leq k_{+} \leq \frac{D}{c_{1, \infty}^{2}} \int\left(\vec{\nabla} c_{1}\right)^{2} \mathrm{~d} V$

for trial functions $c$ satisfying only the boundary conditions at the cell surface.

\section{Type of trial functions}

The reader may have noticed a similarity be. tween the upper and lower bound formalism derived in sections 2 and 3 and the Thomson and Dirichlet bounds on the electrostatic capacitance of a system of conductors [12]. Indeed, the similarity can be made an exact analogy [1-3] by relating the ligand concentration $c$ to the electrostatic potential field $\varphi$ through

$c=c_{\infty}\left(1-\frac{\varphi}{\varphi_{0}}\right)$

where $\varphi_{0}$ is the potential at the surface of the conductor. The receptors are replaced by idea! conductors of identical surface geometry. We have not used this analogy earlier because it implies a loss of generality as the counterpart of the reflecting cell membrane would have to be a dielectric medium with infinite permittivity. Though mathematically sound, the physical significance is not very appealing. For the geometries we shall treat, however, the latter complication can be avoided.

If the radius of curvature of the cell membrane is large compared to the distance over which the concentration profile changes appreciably, we may approximate this surface by a horizontal plane at $z=0$ through which receptors of arbitrary shape project into the plasma. The boundary condition $\partial c / \partial n=0$ at the membrane is satisfied automatically by symmetry arguments if we add to the receptors their mirror images with respect to the plane $z=0$ and solve $\Delta c=0$ for all $z$. In this case we are dealing with a boundary value problem of the Dirichlet type.

In the electrical analogy the relevant entity to be calculated is the capacitance $C$ of the collection of co.nductors, which is related to the diffusionlimited flux

$J=\frac{D c_{\infty}}{2 \epsilon_{0}} C$

where $\epsilon_{0}$ is the permittivity of a vacuum $[1,3]$. The extra factor of 2 appears because for $J$ we are concerned only with the receptors, while $C$ includes their mirror images. In the electrostatic 
setting we then have bounds on the capacitance

$$
\frac{Q^{2}}{2 U[\sigma]} \leq C \leq \frac{2 U[\varphi]}{\varphi_{0}^{2}}
$$

Here $Q$ is the total charge and $\sigma$ the charge distribution. $U[\sigma]$ and $U[\varphi]$ are the electrostatic energy in terms of the charge distribution $\sigma$ and the field $\varphi$, respectively. Written as a functional of the field, the expression for the energy is [13]

$$
U[\varphi]=\frac{\epsilon_{0}}{2} \int(\vec{\nabla} \varphi)^{2} \mathrm{~d} V
$$

This shows that the right-hand side of eq. 22 is equivalent to eq. 9 for the flux $J_{1}$. The total charge $Q$ is defined by

$Q=\int \sigma \mathrm{d} V=-\epsilon_{\mathrm{o}} \int \Delta \varphi \mathrm{d} V=-\epsilon_{\mathrm{o}} \int_{\mathrm{a} V_{\mathrm{o}}} \vec{\nabla} \varphi \cdot \overrightarrow{\mathrm{d}} \overrightarrow{\boldsymbol{S}}$

where we have used Poisson's equation and Gauss' theorem again to derive a form in which the equivalence with $J_{0}$ is more obvious. One observes that the charge can reside only at the boundaries of $V$. Indeed, eq. 22 for $C$ is equivalent to the analogous eq. 18 for the diffusive flux $J$. The point is that this enables us to use the familiar results from electrostatics, which through the analogy [20] can be translated to the equivalent problem in chemoreception. Instead of taking different concentration profiles $c_{1}$ and $c_{2}$ one considers a trial potential $\varphi$, for which one calculates the electrostatic energy to obtain an upper bound on $C$, and a trial charge distribution that when the energy functional is evaluated yields a lower bound.

As a further simplification we take all receptors to be hemispheres of radius $c$, which become spherical conductors if we use the electrostatic analogy. As a trial potential for the collection of spheres we suggest, following Goldstein and Wiegel [3],

$\varphi=\max \left(\varphi_{1} \ldots \varphi_{n}\right)$

with $\varphi_{r}=a \varphi_{0} / r_{r}$ being the potential field of sphere

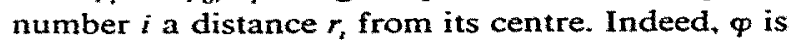
continuous, with some kink discontinuities, but $\bar{\nabla} \varphi$ is always square integrable. For a single sphere, of course, this yields the exact result

$C=4 \pi \epsilon_{0} a$

which leads to the familiar expression for a spherical, completely absorbing cell

$\alpha=\frac{J}{D c_{\infty} a}=\frac{C}{\epsilon_{0} a}=4 \pi$

where $\alpha$ is a dimensionless constant which depends only on the specific geometry of the problem. For a hemispherical receptor $\alpha=2 \pi$.

To calculate lower bounds on $C$ we propose a trial charge distribution

$\sigma_{s}=\frac{q_{i}}{4 \pi a^{2}}$

i.e., a homogeneous distribution of charge on each sphere. If allowed by symmetry arguments we can take $q_{t}=q$, otherwise it may be possible to minimize the energy by varying $q_{t}$, while keeping $Q=$ $\sum q$, fixed. The energy for a collection of spheres splits into a self-energy part of the isolated spheres and an interaction part. Because we know $C$ for one sphere, the self-energy part is given by eq. 26 . The interaction energy, because of the spherical symmetry, is simply that between equivalent point charges situated in the centre of the spheres. This gives

$$
U=\sum_{i=1}^{n} \frac{q_{i}^{2}}{8 \pi \varepsilon_{0} a}+\sum_{i=1}^{n-1} \sum_{j=i+1}^{n} \frac{q_{i} q_{j}}{4 \pi \epsilon_{0} r_{i j}}
$$

where $r_{i f}$ is the distance between the centres of spheres $i$ and $j$. Eq. 29 breaks down when $r_{i}, 2 a$ for any two spheres, as the interaction energy would then be overestimated. The lower bound trial function (eq. 28) is thus somewhat less general than that (eq. 25) for the upper bound estimate.

\section{Specific geometries}

As a first example let us investigate the case of two identical spheres with their centres a distance $b$ apart. For this configuration the exact result is 
known [13]

$$
C=8 \pi \epsilon_{0} a \sinh (\beta) \sum_{n=1}^{\infty} \frac{(-1)^{n+1}}{\sinh (n \beta)}
$$

where $\cosh (\beta)=b / 2 a$. The upper bound estimate in this case, following eq. 25 , yields

$$
C \leq 8 \pi \epsilon_{0} a\left(1-\frac{a}{2 b}\right)
$$

which approaches the exact result for $b \gg a$. The integration which has to be performed to obtain this result, or generally for any planar collection of spheres, is described in detail in appendix $B$. The lower bound estimate, using eq. 29 , is

$c \geq 8 \pi \varepsilon_{0} a\left(1+\frac{a}{b}\right)^{-1}$

which also approaches the exact result for the case $b \gg a$. Indeed, for large $b$ eqs. 31 and 32 approach quite closely. As $1 /(1+x)>(1-x)$ for $x>-1$, the difference between them is less than $a / 2 b$. The maximal difference is obtained for the limiting case $b=2 a$ when the spheres touch. In terms of $\alpha$ we obtain

$\frac{x}{3} \leq \frac{\alpha}{4 \pi} \leq \frac{3}{4}$

while the exact result is $\alpha / 4 \pi=\log 2=0.6931$. The upper bound is $8 \%$ too high, the lower $4 \%$ in error (fig. 2).

For the case of $n$ touching spheres on a strajght line (fig. 4a) we can immediately find the upper bound by noting that the introduction of a third sphere through eq. 25 only affects the field of the second sphere. Eq. 31 then generalises to

$C \leq 2 \pi(n+1) \epsilon_{0} a$

A lower bound on $C$ can be found by application of eq. 29 with all charges equal. We obtain the energy

$$
\begin{aligned}
V & =\frac{q^{2}}{8 \pi \epsilon_{0} a}\left[n+\sum_{i<j} \frac{1}{(j-i)}\right] \\
& =\frac{q^{2}}{8 \pi \epsilon_{0} a}\left[n+\frac{n-1}{1}+\frac{n-2}{2}+\ldots+\frac{1}{n-1}\right] \\
& =\frac{n q^{2}}{8 \pi \epsilon_{0} a}\left[\sum_{k=1}^{n} \frac{1}{k}\right]
\end{aligned}
$$

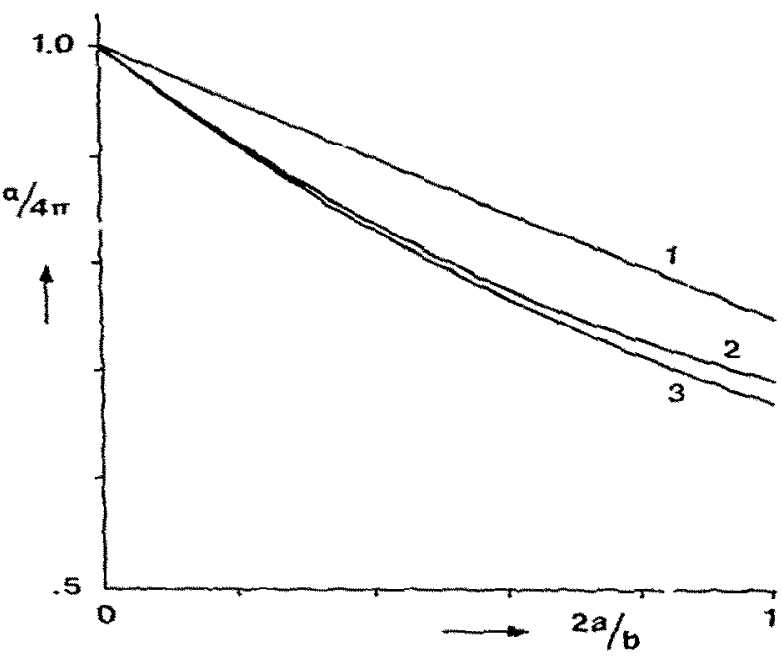

Fig. 2. The geometry factor $\alpha=J / D a c_{x}$ for two hemispheres of radius $a$, whose centres are a distance $b$ apart. Upper bound (1) according to eq. 31 , the exact result (2) according to eq. 30 and the lower bound (3) according to eq. 32. The dumbbell $(b=2 a)$ may be used as a model for the immunoglobulir receptor.

With $Q=n q$, the result for $C$ is

$C \geq 4 \pi \varepsilon_{0}$ an $\left[\sum_{i}^{n} \frac{1}{k}\right]^{-1}$

Thus, we find for $\alpha=C / 2 \epsilon_{0} a$

$\left[\sum_{1}^{n} \frac{1}{k}\right]^{-1} \leq \frac{\alpha}{2 \pi n} \leq \frac{1}{2}\left(1+\frac{1}{n}\right)$

For large $n$ the finite surn may be approximated by

$\sum_{1}^{n} \frac{1}{k}<\gamma+\log n+\frac{1}{2 n}$

with $\gamma=0.5772$ - Euler's constant. One should be careful to include the last term to avoid underestimating the energy. In fact, the other coefficients in the Laurent series in $n$ are all negative [14], which proves eq. 38. This shows that the lower bound in eq. 37 decreases as $1 / \log (n)$ as the number of spheres approaches infinity; the upper bound, however, becomes constant. This dis- 
crepancy is due to the fact that the energy in eq. 34 is greatly overestimated, because the trial function eq. 25 , decreases much too fast near the conductors, giving too high a $\nabla \varphi$.

One needs a better trial function, and here eq. 11 may be of use. It may be observed that $n$ touching unit sphercs in a row can be enclosed by a spheroid of semi-axis $n$ and $\sqrt{n}$, respectively (see appendix A). The capacitance of an ellipsoid is [13]

$C=8 \pi \epsilon_{0}\left\{\int_{0}^{\infty}\left[\left(\alpha^{2}+t\right)\left(\beta^{2}+t\right)\left(\gamma^{2}+t\right)\right]^{-\frac{1}{2}} \mathrm{~d} t\right\}^{-t}$

where $\alpha, \beta$ and $\gamma$ are the semi-axes. For $\alpha>\beta=\gamma$ the integration can be performed analytically, with the result

$$
\begin{aligned}
C & =8 \pi \epsilon_{0} \alpha \sqrt{1-\frac{\beta^{2}}{\alpha^{2}}}\left[\log \left(\frac{1+\sqrt{1-\beta^{2} / \alpha^{2}}}{1-\sqrt{1-\beta^{2} / \alpha^{2}}}\right)\right]^{-1} \\
& =4 \pi \epsilon_{0} \alpha g\left(\frac{\beta^{2}}{\alpha^{2}}\right)
\end{aligned}
$$

If we substitute $\alpha=n a, \beta=a \sqrt{n}$ and expand the result for large $n$, we obtain

$$
\begin{aligned}
g(\delta) & =2 \sqrt{1-\delta}\left[\log \left(\frac{1+\sqrt{1-\delta}}{1-\sqrt{1-\delta}}\right)\right]^{-1} \\
& <\frac{2}{\log 4-\log \delta}
\end{aligned}
$$

with $\delta=1 / n$. This implies an upper bound on $\alpha / 2 \pi n$

$$
\frac{\alpha}{2 \pi n} \leq g\left(\frac{1}{n}\right)<\frac{1}{\log (2 \sqrt{n})}
$$

which indeed also decreases logarithmically when $n \rightarrow \infty$. The logarithmic bound in eq. 42 is sharper than that in eq. 37 for $n \geq 10$; the bound $g(1 / n)$ is better for $n>7$ (fig. 3 ).

As a further example of a configuration of binding sites we use our model to compute bounds on the collective $\alpha$ of three identical hemispheres closely packed in an equilateral triangle (fig. 4b). The upper bound, as calculated according to eq.

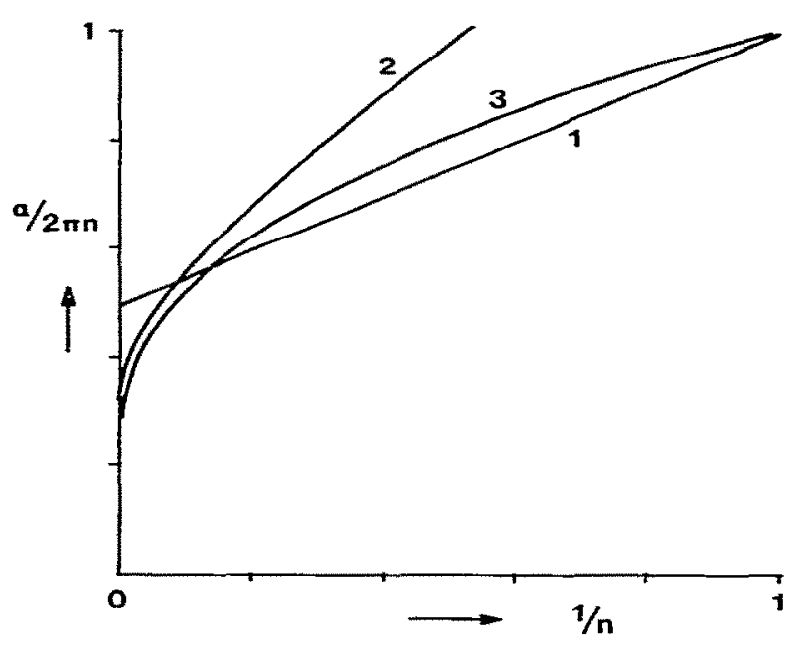

Fig. 3. The reduction of the forward rate constant $k_{+}=\alpha n D a$ for $n$ touching spheres in a row. The exact result for a single sphere is $k_{+}=2 \pi D a$. Upper bound on $\alpha$ as calculated according to eq. 37 , the line (1), and eq. 42 . where $1 / \log (2 \sqrt{n})=(2)$ and $g(1 / n)=(3)$.

\section{5, becomes (appendix B)}

$C \leq \frac{15 \pi \epsilon_{0} a}{2}$

while the lower bound according to eq. 28 with all charges equal yields

$C \geq 6 \pi \epsilon_{0} a$

This gives the result

$\frac{\pi}{2} \leq \frac{\alpha}{6 \pi} \leq \frac{5}{8}$

In the same way, we may consider the case of four closely packed spheres, in a triangular array (fig. 4c)

$\frac{64 \pi \epsilon_{0} a}{9+\frac{1}{3} \sqrt{3}} \leq C \leq 9 \pi \epsilon_{0} a$

or

$0.4176 \leq \frac{\alpha}{8 \pi} \leq \frac{9}{16}$

The lower bound may be improved by lowering the charge on the two middle spheres $q_{2}=q_{3}=(1$ 
$-\delta) q$, while increasing that on the outer two accordingly, i.e., $q_{1}=q_{4}=(1+\delta) q$. Minimisation of $U$ with respect to $\delta$ then yields

$\delta=\frac{\sqrt{3}-1}{\sqrt{3}+1}=0.268$

with the effect that

$\frac{\alpha}{8 \pi} \geq 0.4263$

In the case of a square array (fig. $4 d$ ) the bounds are

$0.4249=\frac{4}{8+\sqrt{2}} \leq \frac{\alpha}{8 \pi} \leq \frac{6-\sqrt{2}}{8}=0.5732$

Here the upper bound can be improved by enclosing the spheres by an oblate spheroid with $\alpha=\gamma=a(1+\sqrt{2}), \beta=\sqrt{a \alpha}$ for which the exact result, using eq. 39 , is

$$
\begin{aligned}
C & =4 \pi \epsilon_{0} \alpha \sqrt{1-\frac{\beta^{2}}{\alpha^{2}}}\left[\arctan \left(\frac{\alpha}{\beta} \sqrt{1-\frac{\beta^{2}}{\alpha^{2}}}\right)\right]^{-1} \\
& \equiv 4 \pi \epsilon_{0} \alpha \mathrm{f}\left(\frac{\beta^{2}}{\alpha^{2}}\right)
\end{aligned}
$$

which results in

$$
\frac{\alpha}{8 \pi} \leq 0.5299
$$

For seven spheres placed in a hexagonal array (fig. 4e), the bounds obtained using eqs. 25 and 29 are

$0.2921=\frac{14}{41+4 \sqrt{3}} \leq \frac{\alpha}{14 \pi} \leq \frac{13}{28}=0.4643$

both of which may be improved: the upper one by considering an oblate spheroid of semi-axis $3 a$ and $a \sqrt{3}$ and the lower by reducing the charge on the central sphere while increasing that on the surrounding ones. The result is

$0.3043 \leq \frac{\alpha}{14 \pi} \leq 0.3663$

It does seem peculiar that the lower bound involves a negative charge on the central sphere, which results in several negative interaction energies through eq. 29 , but this effect is at least partially compensated by an increased self-energy contribution.

In fig. 4 we summarize the bounds that we have obtained on the diffusion-limited forward rate constant $k_{-}=J / c_{\infty}$ for the various hemispherical receptor clusters discussed in this section.

For some of these cltsters, the numerial values we have obtained as bounds on the flux have previously been found by others as approximations. For example, Deutch et al. [10], starting from an electrostatic analogue, showed that the flux intc $n$ spherical sinks of radius $a$ arranged in a

\begin{tabular}{|l|c|c|}
\hline CONEIGURATION & $\begin{array}{c}\text { LOWER } \\
\text { BOUND }\end{array}$ & $\begin{array}{c}\text { UPPER } \\
\text { BOUND }\end{array}$ \\
\hline & $\left(\sum_{k=1}^{n} 1 / k\right)^{-1}$ & $(\log 2 \sqrt{n})^{-1}$ \\
\hline
\end{tabular}

Fig. 4. Compilation of the numerical results obtained for five different planar distributions of he:nispherical receptors. The first column gives the configuration of $n$ spheres when looked upon from a point somewhere above the membrane. The second column gives the reduction for the geometry factor $\alpha=J / D a c_{\infty}$. Tabulated is $\alpha / 2 \pi n$, where $2 \pi n$ would be the result if the receptors were far away from each other. The third column gives the upper bound on $\alpha / 2 \pi n$. 
regular polygon with sides of length $b$ is

$J=4 \pi D a c_{\infty} n\left[1+\left(\frac{a}{b}\right) s(n)\right]^{-1}$

where

$S(n)=\sin \left(\frac{\pi}{n}\right) \sum_{k=1}^{n-1}\left[\sin \left(\frac{\pi k}{n}\right)\right]^{-1}$

For two spheres, eqs. 55 and 56 give the same result as that we obtain as a lower bound in eq. 32 . Similarly, for three identical touching spheres in an equilateral triangle and four touching spheres in a square array, our lower bounds, eqs. 44 and 50, are identical with the approximate solutions of Deutch et àl. [10].

For $n$ identical spheres of radius $a, A$. Szabo (unpublished result) obtained the following approximate solution

$J=4 \pi \operatorname{Danc}_{\infty}\left(1+\frac{2 a}{n} \sum_{i<j} \frac{1}{r_{1},}\right)$

This is equal to the lower bound we obtain when we take all $q_{1}=q$ in eq. 28 .

\section{Discussion}

We have shown how two variational principles may be used to produce both upper and lower bounds on the combined diffusion-limited flux for clusters of chemoreceptors.

For some fairly simple trial functions the results for a collection of hemispherical sites in a planar configuration give quite restrictive bounds. Where exact results are known the bounds can be seen to be remarkably accurate. In the absence of exact results the upper and lower bound for the examples treated differ by about $20 \%$, indicating that the relative error bars in the estimate are of the order of $10 \%$.

For more complex configurations the triai function for the upper bound as proposed was seen to be oversimplified. In the limit of infinitely many receptors even the qualitative result in one case was proven to be wrong. However, the use of the alterrative prescription for a trial function, based on simple geometrical arguments, did give satisfactory results in that case.

The method we used may easily be generalised to three-dimensional cases, provided one refrains from using reflecting boundary conditions. Essentially, this is the only reason why we considered only planar configurations of receptors. Both upper and lower bounds may be calculated for the case of spherical receptors when the ligands can diffuse freely within the configuration.

Even when this is not a reasonable approximation the formalism as developed in sections 2 and 3 is completely general. The only problem is to find a relatively simple trial function that will give nontrivial bounds. When this cannot be done analytically one may of course perform numerical calculations and find rigorous error bars for the exact result with the use of those formulae.

\section{Appendix A: Covering a collection of spheres by a spheroid}

We look for the smallest spheroid that will cover a collcction of spheres of unit radius, whose centres lie in a plane $A$. We consider the plane $B$ perpendicular to $A$, intersecting it along the line connecting the centre of gravity $C_{0}$ of the whole collection and the centre $C_{1}$ of the most remote sphere. Let the distance between the latter two points be $\beta-1$. The cross-section of this sphere and plane $B$ (fig- 5) may then be parametrized by

$y_{1}=1-\sqrt{1-x^{2}}$,

and the ellipse we seek can be defined as

$y_{2}=\beta-\beta \sqrt{1-x^{2} / \alpha^{2}}$

We now set $y_{1} \geq y_{2}$ for $0 \leq x \leq 1$ in order to determine $\alpha$. A Taylor expansion of the square root

$\sqrt{1-\epsilon}=1-\sum_{n=1}^{\infty} a_{n} \epsilon^{n}$

converges for $0 \leq \epsilon \leq 1$, where it is sufficient to note that $a_{n}>0$ for all $n$. This gives the condition

$\sum_{1}^{\infty} a_{n} x^{2 n} \geq \beta \sum_{1}^{\infty} a_{n}\left(\frac{x}{\alpha}\right)^{2 n}$

which is satisfied provided $\alpha^{2} \geq \beta \geq 1$. 


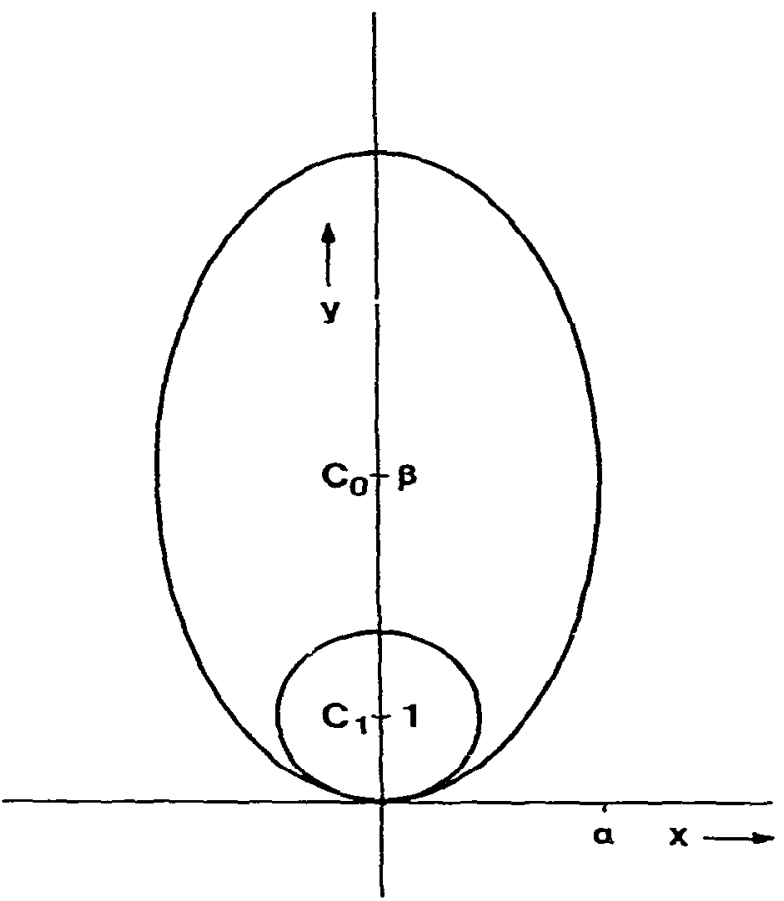

Fig. 5. Cross-section of the sphere whose centre $C_{t}$ is most remote from the centre $C_{0}$ of a collection of unit spheres, and the spheroid covering the whole collection.

\section{Appendix B: Integration over a triangle configura- tion.}

We consider three identical spheres of radius $s$ that are placed in a triangular configuration (fig. 6). Evaluation of the trial potential (eq. 25) involves integration over triangular-shaped regions, like the lightly hatched area, extending to infinity perpendicularly to the plane of the paper, and semi-infinite slabs, like the heavily hatched region. If the centre of the relevant sphere is taken as the origin of a spherical coordinate system, the general expression for such an integral is

$$
\begin{aligned}
U= & \frac{\epsilon_{0}}{\varphi_{0}^{2}} \int_{0}^{\varphi} \mathrm{d} \varphi \int_{0}^{\pi} \mathrm{d} \theta \int_{s}^{r / 2 \cos \varphi \sin \theta} \\
& \times \mathrm{d} r\left[r^{2} \sin \theta\left(\frac{s \varphi_{0}}{r^{2}}\right)^{2}\right]
\end{aligned}
$$

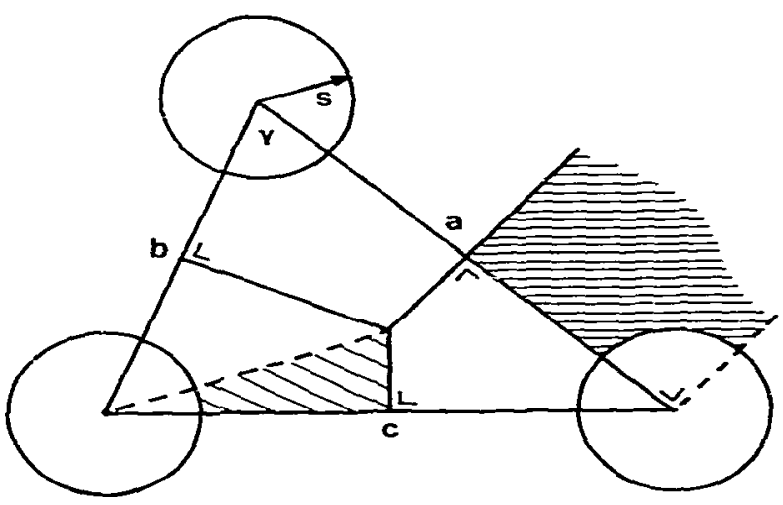

Fig. 6. Planar configuration of three identical spheres of radius 5. The lightly and heavily hatched areas are the cross-sections of the plane with the regions over which the integration in eq. B1 must be performed.

The integration may be performed and yields

$$
u=2 \epsilon_{0} s\left[\varphi-\frac{\pi s}{2 r} \sin \varphi\right]
$$

For the lightly shaded triangle $p=(\pi / 2)-\gamma$ and $r=c$, with the result

$u_{1}=\epsilon_{0} s\left[\pi-2 \gamma-\frac{\pi s}{c} \cos \gamma\right]$

while the darkly shaded slab with $p=\pi / 2$ and $r=a$ gives

$u_{2}=\pi \epsilon_{0} s\left[1-\frac{s}{a}\right]$

Now all space is covered by regions which contribute energies of the type $u_{1}$ or $u_{2}$, except for three separate volumes the contribution of which can be seen to combine to give the energy of a single sphere

$u_{0}=4 \pi \epsilon_{0} s$

Combination of all the $u_{1}$ terms using the cosine rule yields

$U_{1}=2 \pi \epsilon_{0} s\left[1-s\left(\frac{a^{2}+b^{2}+c^{2}}{2 a b c}\right)\right]$

while addition of all $u_{2}$ terms gives

$U_{2}=2 \pi \epsilon_{0} s\left[1-s\left(\frac{1}{a}+\frac{1}{b}+\frac{1}{c}\right)\right]$ 
The total energy of the trial potential field for this configuration becomes

$$
U=12 \pi c_{0} s\left[1-s \frac{(a+b+c)^{2}}{12 a b c}\right]
$$

which for $a=b=c=2 s$ indeed may be seen to lead to the result, eq. 43 . Note that the result for an obtuse triangle will be the same, due to the fact that formally one of the angles will give a negative contribution $u_{1}$.

\section{Acknowledgements}

The work of J.H.J. v. O. is part of the research program of the Stichting voor Fundamenteel Onderzoek der Materie (F.O.M.), which is financially supported by the Nederlandse Organisatie voor Zuiver Wetenschappelijk Onderzoek (Z.W.O.). The work of B.G. has been performed in part under the auspices of the Department of Energy and is supported by grant Al16465 from the National Institute of Allergy and Infectious Disease.

\section{References}

1 H C. Berg and E.M. Purcell, Biophys, J. 20 (1977) 193.

2 F.W. Wiegel. Phys. Rep. 95 (1983) 283.

3 B. Goldstein and F.W, Wiegel, Biophys. J. 43 (1982) 121.

4 M.V. Smoluctiowski, Phys. Z. 17 (1916) 585.

5 L. Jarett, R.M.. Schweitzer and R.M. Sr:zith, Science 210 (1980) 1127.

6 J.L. Goldstein, R.G.W. Anderson and M.S. Brown, Nature 279 (1979) 679.

7 J.-L. Carpentier, P. Gorden, R.G.W. Anderson, J.L. Goldstein, M.S. Brown, S. Cohen and L. Orci, J. Cell Biol. 95 (1982) 73 .

8 A. Nisonoff, J.E. Hopper and S.B. Spring. The antibody molecule (Academic Press, 1975, New York) 2-3.

9 D. Wattens and A. Maelicke, Biochemistry 22 (1983) 1811.

10 J.M. Deutch, B.U. Felderhof and M.J. Saxton, J. Cherr. Phys. 11 (1976) 4559.

11 A.M. Arthurs. Complementary variational principles, (Clarendon Press, 1980, Oxford).

12 R. Courant and D. Hilberh, Methods of Mathematical Physics, Vol. I (Interscience, 1953, New York).

13 W.R. Smythe, Static and dynamic electricily (McGraw-Hill. 1968, New York).

14 I.S. Gradshteyn, and P.M. Ryzhik. Table of integrals, series, and products (Academic Press, 1965, New York) form. 0.131 . 